\title{
ALTERAÇÕES DAS PARTÍCULAS DE AMIDO DE MILHO AGLOMERADAS COM ALGINATO DE CÁLCIO PELO MÉTODO "DRIPPING"
}

\author{
G. FELTRE, ${ }^{1,2 *}$, F. C. MENEGALLI ${ }^{2}$, G. C. DACANAL ${ }^{1}$ \\ ${ }^{1}$ Universidade de São Paulo, Faculdade de Zootecnia e Engenharia de Alimentos, Dep. \\ Engenharia de Alimentos \\ ${ }^{2}$ Universidade Estadual de Campinas, Faculdade de Engenharia de Alimentos, Dep. Engenharia de \\ Alimentos \\ *e-mail: gabifeltre@hotmail.com
}

\begin{abstract}
RESUMO
O amido de milho é uma importante fonte de energia para os seres humanos e é muito utilizado em preparos de diversos pratos. $\mathrm{O}$ amido nativo possui estrutura semicristalina que sofre degradação em elevadas temperaturas e com presença de água. Com o objetivo de alterar a microestrutura do amido, principalmente quanto à temperatura de gelatinização, foi realizada a aglomeração de partículas utilizando-se alginato de sódio e cloreto de cálcio, pelo método de gotejamento, ou dripping. As partículas produzidas foram analisadas por DSC, FT-IR, DRX e MEV. Foram obtidas partículas com diferentes composições, mais especificamente, em frações de $(0,50,60,70,80$ e 90)\% de amido. As modificações ocorreram à medida em que se aumentou a quantidade de alginato de sódio da partícula. As partículas com menores frações de amido, ou seja, maiores conteúdos de alginato de cálcio, apresentaram pequenas alterações em sua microestrutura e cristalinidade, mas tiveram picos endotérmicos em temperaturas mais elevadas.
\end{abstract}

\section{INTRODUÇÃO}

$\mathrm{O}$ amido é a principal fonte de energia ao redor do mundo (SINGH et al., 2010). Seus grânulos possuem natureza semicristalina e a fração de amilose e amilopectina presentes variam de acordo com a espécie botânica, determinando seu grau de cristalinidade (SINGH et al., 2003).

Os grânulos de amido podem ser classificados quanto a sua digestibilidade. $\mathrm{O}$ amido de rápida digestão é digerido após 20 minutos de sua ingestão e fornece energia rapidamente ao organismo. $\mathrm{O}$ amido de lenta digestão apresenta tempo de digestão entre 20 e 120 minutos após a sua ingestão, fornecendo energia lentamente e mantendo a glicose no sangue. Sua digestão ocorre no intestino delgado. $\mathrm{O}$ amido resistente é digerido após 120 minutos de sua ingestão e não ocorre no intestino delgado; sua ação ocorre no cólon (LEHMANN; ROBIN, 2007). $\mathrm{O}$ amido resistente destaca-se por não ser digerido pelas enzimas do corpo humano (SAJILATA et al., 2006) atuando semelhante a uma fibra solúvel no cólon humano (HARALAMPU, 2000).

A aglomeração consiste na modificação de tamanho de partículas, decorrente da união das mesmas na presença de um líquido ligante, para a formação de uma estrutura maior chamada de agregado poroso, que se trata de uma partícula maior (DACANAL; MENEGALLI, 2009). O processo de aglomeração de partículas é muito utilizado em alimentos particulados para alteração de suas propriedades, como fluidez e solubilidade (TURCHIULI et al., 2005). 
Para a encapsulação de compostos ativos, como enzimas e drogas, dentro de uma matriz, um método muito utilizado é o “dripping”. Nessa técnica, uma solução é extrudada através de um capilar a uma vazão baixa e cai em outra solução por gravidade. Assim, é possível recobrir uma partícula com alginato de cálcio, como estudou Chan et al. (2009).

O método "dripping" é um dos mais antigos e mais simples métodos para microencapsulação de compostos. Existem diversos tipos de "dripping". Três deles são apresentados na Figura 1 (WHELEHAN; MARISON, 2011).

Figura 1 - Três diferentes métodos de "dripping". (a) "dripping" simples; (b) "dripping" com extrusão eletrostática; (c) "dripping" com fluxo de ar coaxial (WHELEHAN; MARISON, 2011).

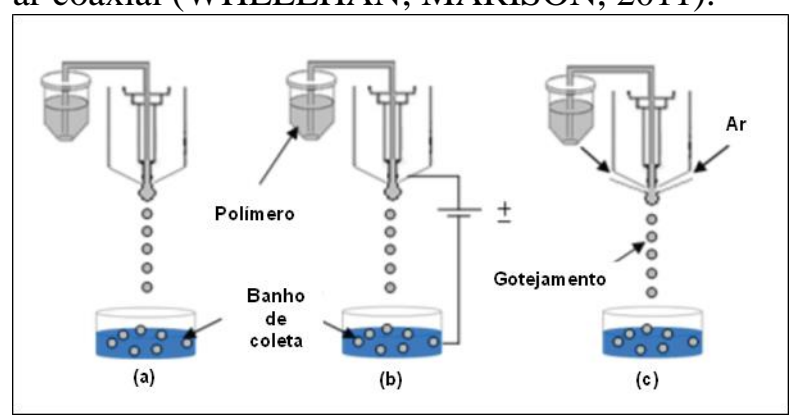

Com o objetivo de gerar uma barreira física em aglomerados de grânulos de amido de milho, para que se apresentassem mais resistentes à gelatinização do que grânulos de amido comuns, foi realizado o processo de aglomeração de grânulos de amido de milho com alginato de cálcio, a partir do método "dripping".

Para análise das modificações interparticulares ocorridas nos grânulos de amido de milho, foram realizadas análises para verificação do aumento da temperatura de gelatinização e modificações nas ligações e estruturas cristalinas presentes nos grânulos.

\section{MATERIAIS E MÉTODOS 2.1 Materiais}

Para a produção das partículas aglomeradas de amido de milho e alginato de cálcio foram utilizados amido de milho (Ingredion Brasil - Ingredientes Industriais Ltda, Brasil), solução de alginato de sódio a $3,5 \%$ (w/w) (Protanal VK14, FMC, EUA), álcool etílico (Dinâmica, mín. 99,5\%, Brasil) e solução de cloreto de cálcio dihidratado $\mathrm{P}$. A. a $1 \%(w / v)$ (Sigma-Aldrich, EUA).

\subsection{Métodos}

\subsubsection{Produção das partículas}

Primeiramente, foi preparada uma solução de alginato de sódio a 3,5\% (w/w). Para melhor dispersão e solubilização em água, e sem formação de grumos, adicionouse cerca de $1 \mathrm{ml}$ de álcool etílico ao pó de alginato. Posteriormente, foram adicionadas quantidades diferentes de amido de milho para cada um dos 6 experimentos realizados, para que as partículas possuíssem determinadas frações de amido depois de secas. As amostras foram nomeadas conforme sua concentração de amido de milho, como é apresentado na Tabela 1.

Tabela 1 - Composição das partículas de amido de milho em cada um dos experimentos realizados.

\begin{tabular}{ccccc}
\hline Amostra & $\begin{array}{c}\text { Fração de } \\
\text { Amido } \\
(\boldsymbol{\%})\end{array}$ & $\begin{array}{c}\text { Amido } \\
(\mathbf{g})\end{array}$ & $\begin{array}{c}\text { Água } \\
(\mathbf{g})\end{array}$ & $\begin{array}{c}\text { Alginato } \\
(\mathbf{g})\end{array}$ \\
\hline SA00 & 0 & 0 & 160,83 & 5,83 \\
SA50 & 50 & 6,69 & 160,83 & 5,83 \\
SA60 & 60 & 10,03 & 160,83 & 5,83 \\
SA70 & 70 & 15,6 & 160,83 & 5,83 \\
SA80 & 80 & 26,76 & 160,83 & 5,83 \\
SA90 & 90 & 60,21 & 160,83 & 5,83 \\
\hline
\end{tabular}

As suspensões foram mantidas sob agitação mecânica a $700 \mathrm{rpm}$, e bombeadas por um tubo de $6 \mathrm{~mm}$ até um bico gotejador, constituído de uma agulha para medicamentos $(0,70 \mathrm{~mm}$ de diâmetro interno) e um tubo de 
silicone para a passagem de ar comprimido paralelamente ao sentido da formação das gotas. Desta forma, a agulha utilizada e o tubo de silicone foram acoplados, formando dois tubos concêntricos, onde no centro (agulha) ocorreu o escoamento da suspensão, e no ânulo ocorreu a passagem de ar comprimido. A vazão de solução ligante foi mantida em 0,5 $\mathrm{mL} / \mathrm{min}$, e a vazão de ar comprimido foi ajustada manualmente, mantendo-se a válvula reguladora de pressão em 1,5 bar. As gotículas produzidas pelo bico foram depositadas em solução aquosa de cloreto de cálcio a $1 \%(w / v)$, e sob agitação magnética. As condições operacionais resultaram em um sistema de gotejamento que produziu partículas individualmente. $\mathrm{O}$ esquema da produção das partículas é apresentado na Figura 2.

Figura 2 - Esquema da produção das partículas aglomeradas de amido de milho e alginato de cálcio.

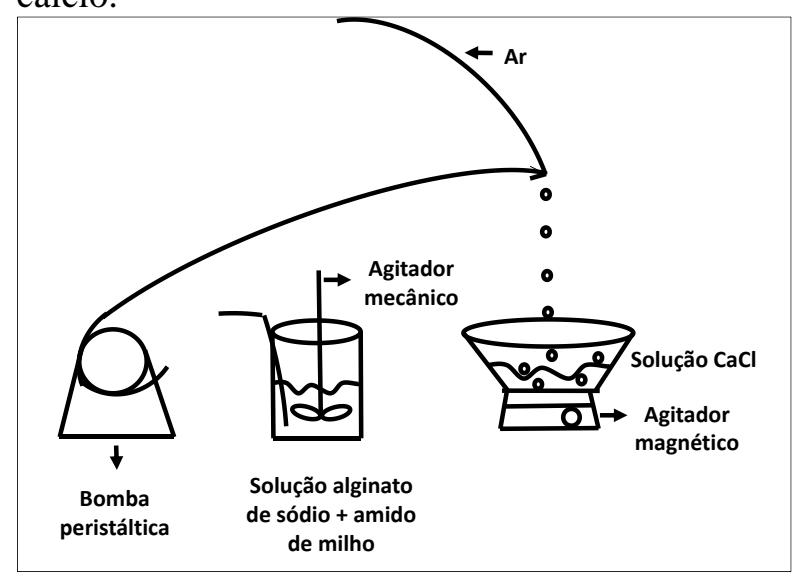

As partículas produzidas foram retiradas da solução de cloreto de cálcio, colocadas em placas de Petri e secas em estufa convectiva a $65^{\circ} \mathrm{C}$ por 24 horas.

\subsubsection{Calorimetria Diferencial de Varredura (DSC)}

Para a realização das análises de calorimetria diferencial de varredura foi utilizado um equipamento de DSC TA 2010 controlado por módulo TA 4000 (TA
Instruments, EUA) e com acessório para resfriamento (SOBRAL et al., 2001).

Uma pequena amostra, de aproximadamente $6 \mathrm{mg}$, das partículas aglomeradas foi acondicionada em uma cápsula e adicionou-se água destilada para se obter uma suspensão de aproximadamente $80 \%$ de água. O tempo de hidratação das amostras foi de 2 horas. As amostras foram aquecidas de $20{ }^{\circ} \mathrm{C}$ a $120{ }^{\circ} \mathrm{C}$ a uma taxa de 10 ${ }^{\circ} \mathrm{C} / \mathrm{min}$. Transições térmicas de gelatinização foram caracterizadas por To (temperatura inicial), $\mathrm{Tp}$ (temperatura do pico endotérmico), Tc (temperatura de conclusão) e $\Delta \mathrm{H}$ (entalpia de gelatinização) (HAN e BEMILLER, 2007; PEREIRA et al., 2010).

2.2.3 Espectroscopia de Infravermelho com Transformada de Fourier (FT-IR)

Para a realização das análises de FT-IR, conforme Vicentini et al (2005), as amostras foram colocadas sobrepostas sob o sensor do espectrofotômetro Spectrum One (Perkin Elmer, Estados Unidos. Foram realizadas 16 varreduras espectrais de 40 a $4.000 \mathrm{~cm}^{-1} \mathrm{com}$ resolução de $2 \mathrm{~cm}^{-1}$. Depois de coletados, os dados foram tratados com o software Spectrum One, (Spectrum One, versão 5.3).

\subsubsection{Difração de Raio-X}

Para as análises de difração de Raio-X foi utilizado o equipamento MiniFlex 600 XRD (Rigaku, Japão). A faixa de ângulo utilizado na análise variou entre $5^{\circ}$ e $60^{\circ} \mathrm{com}$ velocidade de $15 \% \mathrm{~min}$.

Como as partículas aglomeradas encontravam-se com estruturas de esferas grandes, foi necessária uma etapa de redução de tamanho, em que as partículas foram trituradas em almofariz, obtendo-se um pó fino. $\mathrm{O}$ equipamento utilizado permite a análise de DRX apenas para pós. 
2.2.5 Microscopia Eletrônica de Varredura (MEV)

As amostras foram submetidas a análises de microscopia eletrônica de varredura (MEV), a fim de avaliar possíveis transformações na superfície dos grânulos.

$\mathrm{O}$ equipamento utilizado foi $\mathrm{O}$ microscópio eletrônico Hitachi modelo TM3000 (Hitachi, Japão). A fonte de elétrons utilizada no MEV é filamento de tungstênio e a voltagem de $15 \mathrm{kV}$. O equipamento apresenta baixo vácuo e não possui captador de elétrons secundários. Foram utilizados aumentos de até 1,5 mil vezes.

\section{RESULTADOS E DISCUSSÃO}

\subsection{Produção das partículas}

As partículas aglomeradas foram produzidas pelo gotejamento da suspensão de alginato de sódio e amido de milho, em solução de cloreto de cálcio, e apresentaramse individualmente, uma a uma.

Observou-se que quanto menor foi a quantidade de amido, mais circular apresentou-se a partícula. Observou-se que as partículas SA90 foram facilmente quebradas quando manuseadas, diferentemente das partículas com maior concentração de alginato (SA50, SA60 e SA70), as quais apresentaram estrutura mais rígida $\mathrm{e}$ compacta.

A redução da fração de amido e, consequentemente, o aumento da fração de ligante, resultaram no decréscimo do tamanho das partículas aglomeradas, de forma que os grânulos SA90, SA80, SA70, SA60 e SA50 podem ser classificados em tamanhos decrescente, respectivamente.

\subsection{Calorimetria Diferencial de Varredura (DSC)}

Observou-se que o amido de milho de milho nativo apresentou temperaturas de início, pico e final de gelatinização semelhantes com as reportadas na literatura
(SINGH et al., 2003). As partículas aglomeradas com alginato de cálcio SA90, SA80, SA70, SA60 e SA50 apresentaram diferenças em todos os parâmetros.

As partículas SA00 trataram-se de partículas de alginato de cálcio e sem amido em sua composição. Assim, não houve pico de gelatinização para essa amostra dentro da temperatura estipulada, de $20{ }^{\circ} \mathrm{C}$ a $100{ }^{\circ} \mathrm{C}$, como pode ser observado na Figura 3.

Figura 3 - Curvas de DSC das partículas de amido de milho aglomeradas com alginato de cálcio por "dripping", com diferentes concentrações de amido.

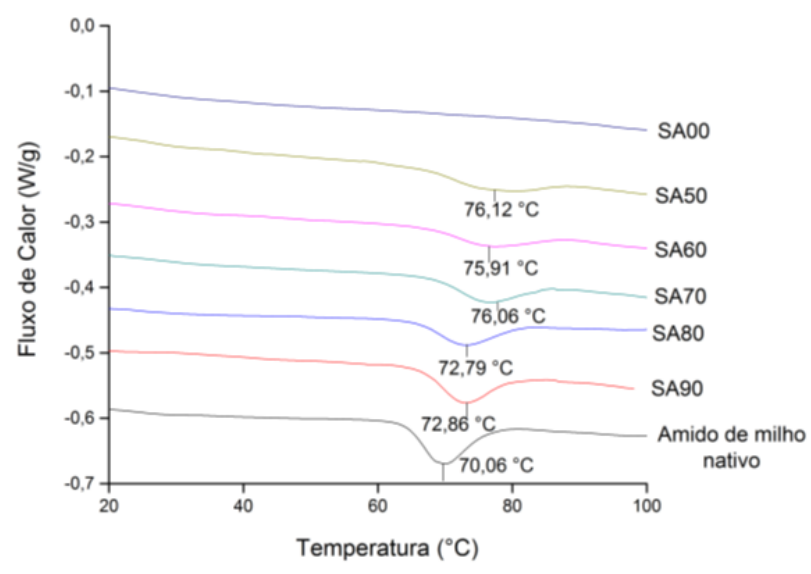

Para as partículas aglomeradas com 90\% de grânulos de amido em sua composição (SA90), os valores de To, Tm e Tc tiveram um aumento de $2{ }^{\circ} \mathrm{C}$, em comparação com o amido de milho nativo. Conforme é apresentado na Figura 3 e na Tabela 2, à medida em que a quantidade de grânulos de amido diminuiu nas amostras, ou seja, que a quantidade de alginato de cálcio aumentou, as temperaturas ficaram gradativamente maiores que as temperaturas do amido nativo.

A entalpia de gelatinização das partículas aglomeradas ficaram menores conforme diminuiu-se a quantidade de amido presente nas partículas. A entalpia das partículas SA50 foi de $6,285 \mathrm{~J} / \mathrm{g}$, um pouco menos da metade da entalpia de gelatinização 
dos grânulos de amido de milho nativo, que foi de $12,602 \mathrm{~J} / \mathrm{g}$. (Tabela 2). Porém, se considerarmos a entalpia de gelatinização pela fração de grânulos de amido de milho presente nas partículas, não há diferença significante entre os $\Delta \mathrm{Hgel}$ do amido de milho nativo e das partículas SA50. Isso porque, se for considerado a entalpia de gelatinização por grama de amido, e não de matéria seca, o valor da entalpia de AS50 é de 12,570 Joules para cada grama de amido.

Tabela 2 - Valores de To, Tm, Tc e $\Delta \mathrm{H}$ das partículas aglomeradas com alginato de cálcio por "dripping", com diferentes concentrações de amido

\begin{tabular}{cccccc}
\hline Amostra & $\begin{array}{c}\text { Umidade } \\
(\%)\end{array}$ & To $\left({ }^{\circ} \mathbf{C}\right)$ & $\begin{array}{c}\text { Tm } \\
\left({ }^{\circ} \mathbf{C}\right)\end{array}$ & $\begin{array}{c}\text { Tc } \\
\left({ }^{\circ} \mathbf{C}\right)\end{array}$ & $\begin{array}{c}\Delta \text { Hgel } \\
(\mathbf{J} / \mathbf{g})\end{array}$ \\
\hline $\begin{array}{c}\text { Amido de } \\
\text { milho }\end{array}$ & & & & & \\
nativo & 78,17 & 64,4 & 70,06 & 80,64 & $12,602^{* *}$ \\
SA90 & 76,28 & 66,82 & 72,86 & 83,91 & $9,616^{* *}$ \\
SA80 & 81,66 & 65,96 & 72,79 & 83,63 & $9,711^{* *}$ \\
SA70 & 74,95 & 68,85 & 76,06 & 86,26 & $6,754^{* *}$ \\
SA60 & 80,98 & 67,95 & 75,91 & 88,03 & $7,040^{* *}$ \\
SA50 & 76,34 & 67,85 & 76,12 & 89,03 & $6,285^{\star *}$ \\
SA00 & 70,36 & $-*$ & $-*$ & $-*$ & $-*$ \\
\hline
\end{tabular}

*Valores não determinados pelo programa.

${ }^{* *}$ Os valores foram calculados baseando-se na massa de amido desidratado.

\subsection{Espectroscopia de Infravermelho com Transformada de Fourier (FT-IR)}

Todos os espectros observados nessa análise apresentaram picos entre (3289 e 3316) $\mathrm{cm}^{-1}$, referentes ao estiramento dos grupos hidroxil (-OH), formadores de pontes de hidrogênio (TAPIA-BLÁCIDO, 2006) e característicos da contribuição da molécula de água, como é apresentado na Figura 4.

A respeito do pico $2926 \mathrm{~cm}-1$ do amido de milho nativo, houve uma diferenciação nas amostras das partículas aglomeradas. Conforme diminuiu-se a quantidade de amido presente, o pico se tornou cada vez menos intenso. Esse pico é referente ao alongamento das ligações $\mathrm{CH} \quad(\mathrm{CH} 2$ assimétrico)
(MAHECHA， 2009; TAPIA-BLÁCIDO, 2006).

O pico na região de $1411 \mathrm{~cm}^{-1}$ presente no espectro do alginato de cálcio, referente à vibração de alongamento assimétrico do grupo - $\mathrm{COO}$, diminuiu de intensidade gradativamente, à medida em que a quantidade de amido presente nas partículas aumentou. Já o pico de $1336 \mathrm{~cm}^{-1}$ observado no espectro de amido de milho nativo diminuiu de intensidade nos outros espectros, conforme diminuiu-se a quantidade de amido presente (Figura 4).

Observou-se que os picos característicos do amido de milho nativo, como em $995 \mathrm{~cm}^{-1}$ mantiveram-se nos espectros das partículas aglomeradas, embora sua intensidade tenha diminuído com o aumento da fração de alginato de cálcio presente.

Figura 4 - Espectro de FT-IR das partículas de amido de milho aglomeradas com alginato de cálcio por "dripping", com diferentes concentrações de amido de milho.

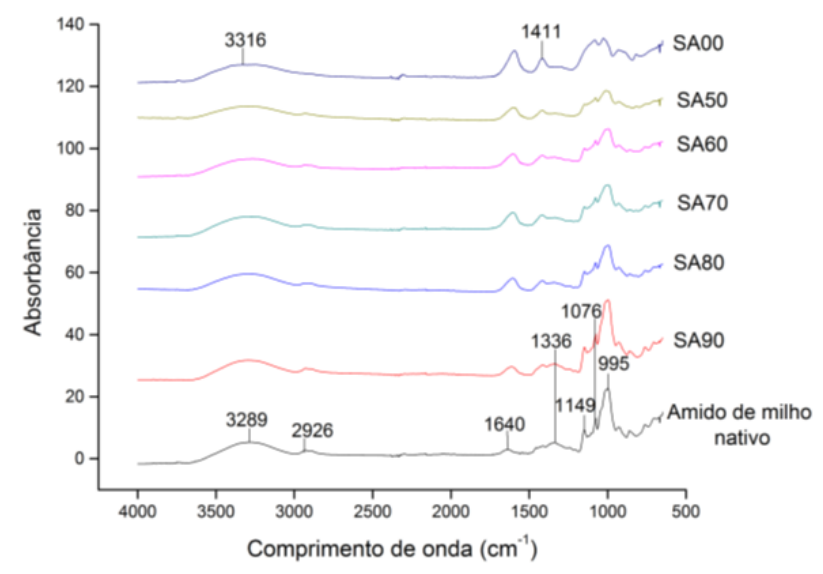

\subsection{Difração de Raio-X (DRX)}

Conforme apresentado na Figura 5, o espectro de DRX de amido de milho nativo apresentou picos em $15^{\circ}$ e $23^{\circ}$ e um pico duplo em $17^{\circ}$ e $18^{\circ}$, característico de domínio cristalino tipo A (PEREIRA et al., 2010). O alginato de cálcio (SA00) apresenta espectro com alguns picos amorfos (ZHAO et al., 2014). 
Observou-se que nos espectros de SA80 e SA90, não houve grandes modificações nos picos, em comparação com o espectro de amido de milho nativo, embora os picos tenham aparecido em menores intensidades. Sugere-se que para essas partículas, houve manutenção do padrão de cristalinidade do amido nativo e que a estrutura foi preservada. As partículas com (80 e 90)\% de amido apresentarm picos relacionados ao alginato de cálcio muito pequenos, como em $11^{\circ}, 25^{\circ} \mathrm{e}$ $29^{\circ}$ (Figura 5).

As partículas SA70, SA60 e SA50 apresentaram picos característicos do alginato de cálcio mais intensos e os picos característicos da cristalinidade do amido pouco intensos.

Os picos referentes à cristalinidade do amido $\left(15^{\circ}, 17^{\circ}, 18^{\circ}\right.$ e $\left.23^{\circ}\right)$ diminuíram de intensidade conforme aumentou-se a quantidade de alginato de cálcio nas partículas. Houve, portanto, maior preservação da estrutura cristalina do grânulo de amido com o aumento da quantidade de grânulos de amido de milho presente.

Figura 5 - Espectro de DRX das partículas de amido de milho aglomeradas com alginato de cálcio por "dripping", com diferentes concentrações de amido.

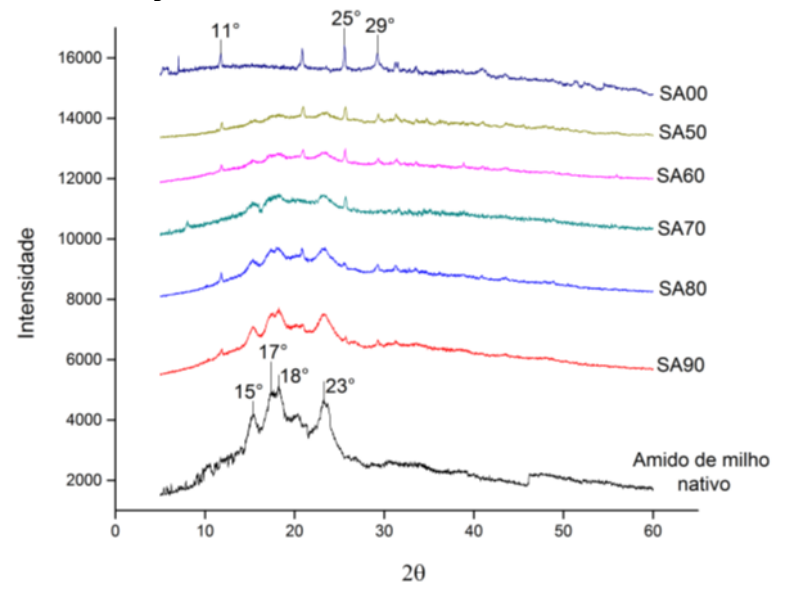

\subsection{Microscopia Eletrônica de Varredura (MEV)}

Observou-se que em relação aos grânulos de amido de milho nativo, as partículas SA90, SA80, SA70, SA60 e SA50 apresentaram-se unidas umas às outras, com a presença de pontes sólidas formadas pelo alginato de cálcio. Fica nítido nas imagens da Figura 6 que conforme a quantidade de amido na partícula foi diminuída, os grânulos de amido encontraram-se mais aglomerados, e com maior número de pontes sólidas visíveis.

Figura 6 - Imagens obtidas por MEV (aumento de 100 vezes) das partículas de amido de milho aglomeradas com alginato de cálcio por "dripping", com diferentes concentrações de amido.
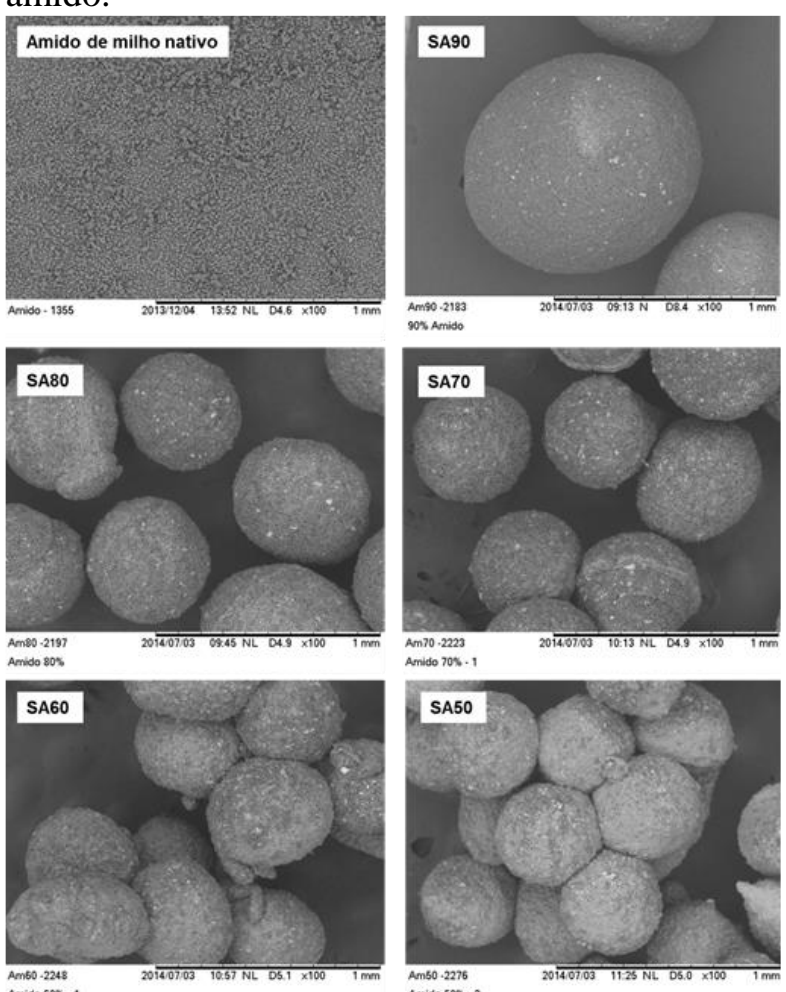
Am70.2223
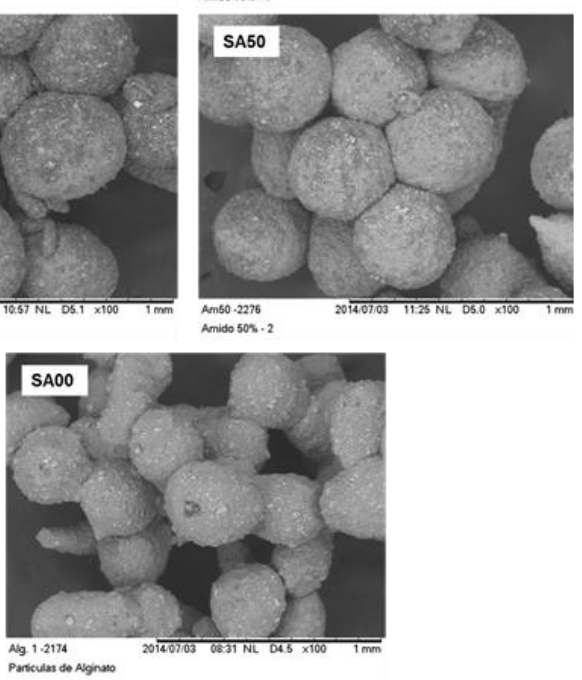
O tamanho das partículas formadas aumentou com adição de amido de milho em sua composição. As partículas com maiores frações de alginato de cálcio, como as SA60 e SA50, apresentaram menores tamanhos. As partículas SA00, sem grânulos de amido de milho, apresentaram os menores tamanhos, além de apresentarem-se unidas umas às outras, devido a pontes formadas durante a secagem convectiva.

\section{CONCLUSÃO}

Diante dos resultados obtidos pelas análises de DSC, observou-se que a aglomeração dos grânulos de amido de milho com o alginato de cálcio pelo método "dripping" gerou partículas com uma estrutura mais resistente à gelatinização. Pode-se, assim, sugerir que a presença do alginato de cálcio nos grânulos de amido de milho criou uma barreira física que proporcionou resistência térmica às partículas aglomeradas.

A partir das análises de MEV, concluiu-se que houve aglomeração das partículas de amido de milho com alginato de cálcio e que elas tornaram-se menores com a diminuição da fração de amido de milho presente.

Por fim, concluiu-se que houve modificações nas partículas produzidas por aglomeração por "dripping" e que elas tornaram-se mais resistentes à gelatinização conforme aumentou-se a fração de alginato de cálcio presente.

\section{REFERÊNCIAS}

CHAN, E.; LEE, B.; RAVINDRA, P.; PONCELET, D. Prediction models for shape and size of ca-alginate produced through extrusion-dripping method. Journal of Colloid and Interface Science, v. 338, p. 6373, 2009.
DACANAL，G. C.; MENEGALLI，F. C. Experimental study and optimization of the agglomeration of acerola powder in a conical fluid bed. Powder Technology, v. 188, p. 187-194, 2009.

HAM, J. A.; BEMILLER, J. N. Preparation and physical characteristics of slowly digesting modified food starches. Carbohydrate Polymers, v. 67, n. 67, p. 366-374, 2007.

HARALAMPU, S. G. Resistant starch - a review of the physical properties and biological impact of RS3. Carbohydrate Polymers, v. 41, p. 285-92, 2000.

LEHMANN U.; ROBIN F. Slowly digestible starch-its structure and health implications: a review. Food Science \& Technology, v. 18, p. 346-355, 2007.

MAHECHA M. M. A. Elaboração e caracterização de biofilms de farinha de biri (Canna indica L.). 2009. 159p. Dissertação (Mestrado em Engenharia de Alimentos) - Faculdade de Engenharia de Alimentos, Universidade Estadual de Campinas, Campinas, 2003.

PEREIRA, N. R.; GODOI, F. C.; ROCHA, S. C. S. Drying of starch suspension in spouted bed with inert particles: physical and thermal analysis of product. Drying Technology, v. 28, p. 1288-1296, 2010.

SAJILATA, M. G.; KINGHAL， R. S.; KULKARNI, P. R. Resistant starch-a review. Comprehensive reviews in food science and food safety, v. 5, p.1-17, 2006.

SINGH, N.; SINGH, J.; KAUR, L.; SODHI, N. S.; GILL B. S. Morphological, thermal and rheological properties of starches from different botanical sources. Food Chemistry, v. 81, p. 219-231, 2003. 
SINGH, J.; DARTOIS, A.; KAUR, L. Starch digestibility in food matrix: a review. Trends in Food Science \& Technology, v. 21, p. 168-180, 2010.

SOBRAL, P. J. A.; MENEGALLI, F. C.; HUBINGER, M. D.; ROQUES, M. A. Mechanical, water vapor barrier and thermal properties of gelatin based edible films. Food Hydrocolloids, v. 15, p. 42-432, 2001.

TAPIA-BLÁCIDO, D. R. Filmes a base de derivados do amaranto para uso em alimentos. 2006. 351p. Tese (Doutorado em Engenharia de Alimentos) - Faculdade de Engenharia de Alimentos, Universidade Estadual de Campinas, Campinas, 2006.

TURCHIULI, C.; ELOUALIA, Z.; ELMANSOURE, N.; DUMOULIN, E. Fluidised bed agglomeration: Agglomerates shape and end-use properties. Powder Technology, v. 157, p. 168-175, 2005.

VICENTINI, N. M.; DUPUY, N.; LEITZELMAN, M.; CEREDA, M. P.; SOBRAL, P. J. A. Prediction of cassava starch edible film properties by chemometric analysis of infrared spectra. Spectroscopy Letters, v.38, n. 6, p. 749-767, 2005.

WHELEHAN, M.; MARISON, I. W. Microencapsulation by dripping and jet break up. Bioencapsulation Innovations, p. 4-10, september, 2011.

ZHAO, K.; FENG, L.; LI, Z.; FU, Y.; ZHANG, X.; WEI, J.; WEI, S. Preparation, characterization and photocatalytic degradation properties of a $\mathrm{TiO} 2 /$ calcium alginate composite film and the recovery of TiO2 nanoparticles. Royal Society of Chemistry, v. 4, p. 51321-51329, 2014.
À CAPES pela bolsa concedida durante o mestrado. À FAPESP e CNPq pelos auxílios financeiros.

\section{AGRADECIMENTOS}

